\title{
Optimal Observer Synthesis for Microgrids With Adaptive Send-on-Delta Sampling Over loT Communication Networks
}

\author{
Seyed Amir Alavi, Graduate Student Member, IEEE, Kamyar Mehran, Senior Member, IEEE, \\ and Yang Hao, Fellow, IEEE
}

\begin{abstract}
State estimation is one of the main challenges in the microgrids, due to the complexity of the system dynamics and the limitations of the communication network. In this regard, a novel real-time event-based optimal state estimator is introduced in this paper, which uses the proposed adaptive send-on-delta (SoD) non-uniform sampling method over wireless sensors networks. The proposed estimator requires low communication bandwidth and incurs lower computational resource cost. The threshold for the SoD sampler is made adaptive based on the average communication link delay, which is computed in a distributed form using the event-based average consensus protocol. The SoD non-uniform signal sampling approach reduces the traffic over the wireless communication network due to the events transmitted only when there is a level crossing in the measurements. The state estimator structure is extended on top of the traditional Kalman filter with the additional stages for the fusion of the received events. The error correction stage is further improved by optimal reconstruction of the signals using projection onto convex sets (POCS) algorithm. Finally, an Internet of things (IOT) experimental platform based on LoRaWAN and IEEE 802.11 (WiFi) protocols is developed to analyse the performance of the state estimator for the IEEE 5 Bus case study microgrid.
\end{abstract}

Index Terms-Event-based state estimation, IoT, LoRaWAN, microgrid, POCS, Real-time simulation, Send-onDelta Kalman filter, Thingsboard.

\section{INTRODUCTION}

Microgrids are small scale power systems that have been proposed for the optimal integration of renewable energy resources (RESs). However, having the smaller scale has decreased the inertia required for the stability of its operation, similar to any other fast dynamic system. Low inertia means that the system is prone to instabilities driven by disturbances more, and therefore robust controllers should be designed to stabilize its long-term operation [1], [2]. The main element of the robust controllers is the adaptation to the current state of the system, as a high number of the robust control techniques are based on state feedback [3], [4]. To address this requirement, observers are used for monitoring the state

Manuscript received June 11, 2020; revised August 06, 2020; accepted October 20, 2020. Authors are with the School of Electronic Engineering and Computer Science, Queen Mary University of London, London, United Kingdom, (e-mail: [s.alavi, k.mehran, y.hao]@qmul.ac.uk). of the system because the state variables of a complex system are not available for direct measurement through the system outputs. Furthermore, due to the measurement noise, process noise, and the communication restrictions, continuous noisefree measurements are not available at the observers, which mandates the usage of estimation strategies in them [5], [6]. Additionally, the fast dynamic of the microgrid necessitates that this estimation takes place in real-time [7].

Two main approaches are proposed in the literature of state estimators, i.e. centralized and distributed strategies. These approaches have their own advantages, which suits them for the specific user application. Distributed state estimation strategies are usually used when the dynamic system has a large scale and the computation resource cost of a centralized option makes the solution expensive and infeasible [8]. Despite distributed approaches solve the single failure point issue, they mandate the use of more measurement nodes, which is not suitable for medium sized microgrids [9], [10].

Contrary to distributed approaches, in centralized state estimators, the main assumption is to have a single node for the whole state estimation task. Therefore, computationally powerful measurement nodes are not needed as they have to only collect the data and transmit it [11], [12]. The data transmission rate of the sensors significantly affects the performance of the estimator, the energy consumption of battery-based devices, and the network traffic [13]. Most of the classic estimation theories have assumed that the signals are either continuous or sampled periodically with a constant period. However, the current communication technology trend is to use Internet of things (IoT) communication networks, which has constraints in the communication speed and its availability [7]. These limitations mandates the use of modern techniques and theories for the state estimation to work based on non-uniform event-based data from the measurements in a microgrid. For example, authors in [14] used non-synchronized measurements from the smart meters, or the authors in [15] have used continuous measurements to find an accurate multilevel estimation strategy. Other studies such as [16]-[18], have tried to reduce the state space set and have used classic estimators, which are not optimal in resource utilization. A data-driven approach is also proposed in [19], which is able to work under uncertainties, with the cost of offline training. Several other works have been reported in the literature to 
address the sampling issue in IoT networks from the rate adaptiveness perceptive. In those works, instead of having the samples at unevenly distributed discrete instants, the periodic sampling rate is adaptively adjusted. In [20], AdaM algorithm is proposed which integrates a filtering unit along with the sampler. This approach increases the computing burden on the device, as it has to solve an optimization problem in realtime. In [21], a differential privacy-based sampling method is proposed, which hides the data correlation from the malicious attacker, with the cost of data encoding/decoding at both the transmitter and receiver ends. A co-design method is proposed in [22], that considers the medium access and sampling rate in the rate adjustment by compressing data into variable sizes chunks. The mentioned works suffer from the high computing burden on the remote monitoring device comparing to eventbased alternatives discussed previously.

The authors proposed an optimal state estimation framework for microgrids using the send-on-delta (SoD) sampling method, in their previous work [23]. The proposed SoD sampling method was delay independent, which only checked if the signal has crossed a predefined threshold as the main condition for the event transmission. Considering the scale of the IoT sensors installed across a microgrid, which can be of thousands or more, this method is an inefficient usage of the communication bandwidth. Also, in that work, the effect of the communication delay was not considered, which is important in IoT wireless sensor networks. Therefore, in this paper to address the the network limitations, computational complexity, and microgrid requirements, a novel solution is proposed that considers the effect of communication delay by adaptively adjusting the threshold of SoD sampler, based on the proposed event-based average consensus protocol. The proposed estimator employs projection onto convex sets (POCS) technique [24] to optimally reconstruct the sparse signals from the measurement nodes and reduces the state estimation error of event-based Kalman filter.

The contributions of this work can be summarized as the proposal of the following items:

- An optimal event-based state estimation framework for microgrids that consists of the proposed event-triggered Kalman filter with the POCS-based signal reconstruction.

- An adaptive threshold SoD sampling method to mitigate the communication delay for accurate state estimation.

- An event-based average consensus protocol for average delay consensus of sampling units such as smart meters.

This paper is organized as follows. In Section II data modelling for both AC and DC microgrids and the architecture of the estimator are discussed. An overview of observer design process is provided in Section III] Then after, the event-based Kalman filter is developed in Section IV] and the POCS signal reconstruction technique is discussed in Section $\mathrm{V}$. The time delay consensus protocol for adaptive threshold SoD sampling is provided in Section VI. The optimality of the solution is analysed from different aspects in Section VII. In Section VIII the results for validation of the observer performance are provided, which is evaluated on a experimental DC microgrid test-bench. The model used for the closed loop control system is derived from our previous work in [1], in which we have proposed a distributed control system for DC microgrids. Finally, the paper is concluded in Section IX

\section{Microgrid State Estimation Data Modelling AND THE OBSERVER ARCHITECTURE}

In this section, the microgrid state estimation problem is modelled from the measurement data viewpoint. A microgrid usually consists of energy storage (ES) systems, renewable energy sources (RESs), consumer loads and power converters. Generally, two voltage systems are considered for microgrids: DC (Direct Current) and AC (Alternating Current) microgrids. Each of these different types are dynamic systems that can be modelled using a set of (non)linear differential equations. Like any other type of dynamical system, every process has internal state variables, outputs, and inputs. The measurements set for the AC and DC microgrids state estimation are \{Active power injected into each bus, Reactive power injected into each bus, RMS voltage of buses\} and \{RMS voltage of buses, RMS of the injected current into each bus $\}$, respectively. Other variables such as phase can be considered for AC microgrids as well, but the phasor measurement units (PMUs) are required for this high speed synchronization, which can be expensive. Therefore, indirect measurements with active and reactive power are used here, which have higher feasibility with lower cost. It is assumed that the measurements from the distributed sensors have the following error dynamics:

$$
\mathbf{z}=\mathbf{h}(x)+\left[\mathbf{e}_{\mathbf{1}} \mathbf{e}_{\mathbf{2}} \ldots \mathbf{e}_{\mathbf{n}}\right]^{\top}
$$

where $\mathbf{z}$ is the output of the sensors, $\mathbf{h}(x)$ is the state to output mapping, and $\mathbf{e}_{\mathbf{i}}$ is the sensor error, which can be due to the noise, or inaccuracy. Also the state dynamics are modelled with the nodal admittance matrix ( $\mathrm{Y}$ bus) of the grid.

The architecture of the proposed state estimator with eventbased measurements is shown in Fig. 1. The three parts are: the event-based adaptive Kalman state estimator, the eventbased signal reconstructor and the mean square error (MSE) comparator. The microgrid estimation input quantities are collected using the proposed adaptive send-on-delta (SoD) measurement technique. The event-based Kalman filter works based on the knowledge that the signal between the events is bounded by the $\delta$ threshold in the SoD sampler. The original signal is reconstructed in the signal conditioner based on the received events using the projection onto convex sets (POCS) algorithm, which is mainly used in the literature as a promising approach for low quality image reconstruction. In the last stage, the error comparator updates the estimated state input based on the difference of the reconstructed signal and the predicted output of the previous filtering stage. The SoD sampler threshold is adapted distributively based on the consensus value of the average communication delay. In this mechanism, each sensor calculates the round trip delay between itself the and the microgrid estimator, and adjusts the threshold according to the fused data from neighbor sensors. The main advantage of this mechanism shows itself when the microgrid components communicate over a shared wireless medium, which is usually the case in IoT-enabled microgrids 


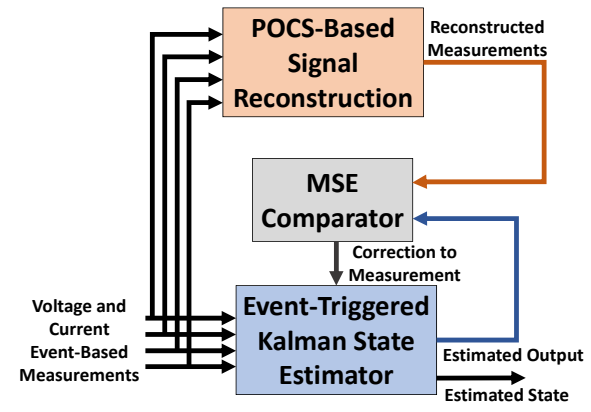

Fig. 1. Proposed event-based structure for microgrid state estimation.

[4]. Optimal usage of network resources, meanwhile providing a high quality estimate of the microgrid state is the aim of the proposed estimation strategy. For example, consider that a microgrid is operating in a transient mode. Usually, in transient modes, the system exposes fast dynamics that lead to a very large number of events using the delay-independent SoD sampling method, which was tackled in our previous work [4]. Communication delay directly proportional to the traffic rate (or packet generation rate) in a shared medium. As a result, the delay on the shared communication medium increases, which considerably decreases the quality of data and the state estimation accuracy. However, in our proposal, if the sensors achieve a consensus on the average communication delay on the shared communication medium, they can automatically adjust the SoD sampling threshold to a wider region, which leads to a lower number of events generated. As a result of this adaptivity, the quality of the data and the state estimation accuracy will be improved considerably, comparing to the previous proposed delay-independent method. To provide this average delay consensus for the sensors in a microgrid, a novel event-based average consensus protocol is proposed in Section VI. which works in parallel with the SoD sampling data flow.

\section{Design Process of The State Estimator}

This section summarizes the steps required for the microgrid state estimator, in a simplified sequence:

1) Finding the global small signal model of the microgrid in the form of a linear state space equation. In this step, the covariance for the process noise and the measurement noise should be chosen according to the microgrid specifications and sensor accuracies. In this work, we have converted the closed loop transfer function matrix, defined in equation (36) of [1], into minimal state space model to get equation (2).

2) Choosing the initial value for the threshold of the $\mathrm{SoD}$ sampler. This value should be selected in accordance with the covariance values chosen in the previous steps, in order to prevent the noisy measurements generate unnecessary events and the resulted traffic.

3) Building the communication topology graph for the event-based average consensus protocol defined in Theorem 1 . The graph should be strongly connected, but doesn't need to be deterministic, as the average consen- sus protocol, designed in the next step, adjusts the SoD threshold dynamically.

4) Choosing the average consensus parameters to have a guaranteed convergence rate for the consensus protocol. This value affects the event generation, therefore a tradeoff takes place between the number of events and the convergence rate of the protocol.

After these steps are taken in the design process, the parameters of the state estimator and the nodes are initialized with the corresponding microgrid parameters. In the results section, the values for the parameters of the case study microgrid are provided together with the results of the experiment. In the next section, the mathematical framework of the event-based Kalman filter with SoD sampling is developed.

\section{Event-Based KaLman Filter based on SEND-ON-DELTA}

Minimal realization of the microgrid admittance bus (Y bus) and the small signal model of the controllers, results in the following multi-variable system for the estimation problem:

$$
\begin{aligned}
& \dot{x}=A x(t)+w(t) \\
& y(t)=C x(t)+v(t)
\end{aligned}
$$

where $x \in \mathrm{R}^{\mathrm{n}}$ is the system state and $y \in \mathrm{R}^{\mathrm{p}}$ is the measured output. $w(t)$ and $v(t)$ are the process noise and measurement noise, respectively, which are the uncorrelated, zero-mean white Gaussian random processes, satisfying the following:

$$
\begin{aligned}
& \mathrm{E}\left\{w(t) w(s)^{\prime}\right\}=Q \delta(t-s) \\
& \mathrm{E}\left\{v(t) v(s)^{\prime}\right\}=R \delta(t-s) \\
& \mathrm{E}\left\{w_{i}(t) v_{j}(s)^{\prime}\right\}=0, \quad 1 \leq i \leq n, \quad 1 \leq j \leq p
\end{aligned}
$$

$R$ is the measurement noise covariance, and $Q$ is the process noise covariance. Also, $w_{i}$ and $v_{j}$ are the $i$-th and $j$-th elements of the $w$ and $v$, respectively. It is presumed that the $i$-th sensor only transmits the data when the difference between the current value and the previous value is greater than the SoD threshold $\delta_{i}$. Using SoD method [25], the estimator continuously samples the data with a period of $T$ from the measurement nodes. For example, if the last received $i$-th sensor value is $y_{i}$ at time $t_{\text {last }, i}$, and there is no data received from $i$-th node for $t>t_{\text {last }, i}$, then $y_{i}(t)$ is estimated as:

$$
y_{i}\left(t_{\text {last }, i}\right)-\delta_{i} \leq y_{i}(t) \leq y_{i}\left(t_{\text {last }, i}\right)+\delta_{i}
$$

The last received $i$-th sensor data is used to compute the output $y_{\text {computed }, i}$ even if there is no sensor data transmission:

$y_{\text {computed }, i}(t)=y_{i}\left(t_{\text {last }, i}\right)=C_{i} x(t)+v_{i}(t)+\Delta_{i}\left(t, t_{\text {last }, i}\right)$

where $\Delta_{i}\left(t, t_{\text {last }, i}\right)=y_{i}\left(t_{\text {last }, i}\right)-y_{i}(t)$ and:

$$
\left|\Delta_{i}\left(t, t_{\text {last }, i}\right)\right| \leq \delta_{i}
$$

In (7), measurement deviation increases from $v_{i}(t)$ to $v_{i}(t)+\Delta_{i}\left(t, t_{\text {last }, i}\right) . \Delta_{i}\left(t, t_{\text {last }, i}\right)$ is assumed to have the uniform distribution constrained by $(8)$, therefore the variance of $\Delta_{i}\left(t, t_{\text {last }, i}\right)$ is $\frac{(2 \times \delta)_{i}^{2}}{12}$, which will be added to the output noise covariance matrix, $\mathrm{R}(i, i)$, in the Kalman estimator. 
SoD-based State Estimation Algorithm: In order to suitably improve the update part of the standard Kalman filter algorithm, an improved algorithm is proposed here, which makes it adapted to the SoD event triggering condition by increasing the input covariance $\bar{R}_{k}$, at the instant of the events:

1) Initialization step

$$
\begin{aligned}
& \hat{x}^{-}(0), P_{0}^{-} \\
& y_{\text {last }}=C \hat{x}^{-}(0)
\end{aligned}
$$

2) Input measurement update

$$
\bar{R}_{k}=R
$$

\section{if $i$-th event are received}

$$
\hat{y}_{\text {last }, i}=y_{i}(k T)
$$

else

$$
\bar{R}_{k}(i, i)=\bar{R}_{k}(i, i)+\frac{(2 \times \delta)_{i}^{2}}{12}
$$

end if

$$
\begin{aligned}
& K_{k}=P_{k}^{-} C^{\prime}\left(C P_{k}^{-} C^{\prime}+\bar{R}_{k}\right)^{-1} \\
& \hat{x}(k T)=\hat{x}^{-}(k T)+K_{k}\left(\hat{y}_{\text {last }}-C \hat{x}^{-}(k T)\right) \\
& P_{k}=\left(I-K_{k} C\right) P_{k}^{-}
\end{aligned}
$$

\section{3) Project ahead}

$$
\begin{aligned}
& \hat{x}^{-}((k+1) T)=\exp (A T) \hat{x}(k T) \\
& P_{k+1}^{-}=\exp (A T) P_{k} \exp \left(A^{\prime} T\right)+Q_{d}
\end{aligned}
$$

where $Q_{d}$ is the covariance of the process noise for the discretized microgrid state space realization, and $y_{\text {last }}$ is defined as follows (15):

$$
y_{\text {last }}=\left[y_{\text {last }, 1}, y_{\text {last }, 2}, \ldots, y_{\text {last }, p}\right]^{\prime}
$$

The presented event-based estimator is also able to be used in the implementation of the distributed controllers in networked systems. For further studies on the convergence analysis, one may refer to [7]. It should be noted that in the proposed event-based observer, convergence is achieved by knowing the fact that Kalman filer is an optimal observer. Nevertheless, choosing smaller values for $\delta_{i}$ would result in the a significant decrease in convergence time [25].

\section{Signal Reconstruction Formulation and ESTIMATOR UPDATE RULE}

The SoD sampled version of a signal contains the time instants that the original signal has changed more than the threshold in the SoD sampling (i.e. $\delta$ ). If no sample has been generated by the SoD sampler, then it means that the original signal has remained in the region around the last event value with the radius of $\delta$. Here, this is called the implicit information in the event data, which is used to solve the optimization problem of signal fitting and reconstruction. To formulate the optimization problem, the solution boundaries need to be determined. The implicit information from the SoD sampled signals are used to determine the required boundaries for the solution of the convex optimization problem, which is modelled in the following. To model and solve the optimization problem, projection onto convex sets (POCS) technique is used, which has been previously used for image reconstruction from low resolution cameras [26], [27] and for signal recovery from level crossing samples [28]. SoD sampling is a generalization of level crossing or Lebesgue sampling, which also considers the signal initial value. To adjust this sampling technique to POCS formulation, the results of level-crossing sampling from [24] are extended, detailed in the next Section.

\section{A. Implicit Information of Send-on-Delta Sampled Signal}

Send-on-Delta sampling is a type of event-based sampling, where each event shows a crossing of the signal $x(t)$ from a one dimensional region bounded by $\delta$ around the last sample. The event time instants $t_{n} \in \mathbb{Z}, n \in \mathbb{Z}$ are defined as:

$$
t_{n}=\min \left\{t>t_{n-1}, x(t)-x\left(t_{n-1}\right)>\delta\right\}
$$

The output of SoD sampler is the sequence of pairs $\left(t_{n}, x\left(t_{n}\right)\right)$. The set of possible samples by assuming zero initial conditions is $X_{e}=\left\{x\left(t_{0}\right), x\left(t_{1}\right), x\left(t_{2}\right), \ldots, x\left(t_{n}\right)\right\}$. In order to formulate the convex optimization problem, a convex region for the possible range of the reconstructed signal is defined according to 16 :

$$
\theta^{-}(t) \leq x(t)<\theta^{+}(t)
$$

where $\theta^{-}(t)$ and $\theta^{+}(t)$ are the piece-wise constant lower and upper boundaries respectively, that are created from the following constraints:

$$
\begin{aligned}
& \theta^{-}(t)=\left\{r \in \mathbb{R}, r=x(k)-\delta, k \in t_{n}\right\} \\
& \theta^{+}(t)=\left\{r \in \mathbb{R}, r=x(k)+\delta, k \in t_{n}\right\}
\end{aligned}
$$

With this definition, the sign of the signal slope at the event instants $\left(t_{n}\right)$ is defined as:

$$
S\left(t_{n}\right)= \begin{cases}x\left(t_{n}\right)-x\left(t_{n-1}\right), & x\left(t_{n}\right) \neq x\left(t_{n-1}\right) \\ S\left(t_{n-1}\right), & x\left(t_{n}\right)=x\left(t_{n-1}\right)\end{cases}
$$

The samples along with the implicit boundary information, take a form of sets membership. Therefore the solution for the reconstructed signal $x(t)$ will fall into the following convex sets $\left(C(\mathbb{R})\right.$ and $\mathbb{L}^{2}$ denote continuous function and Hilbert space, respectively):

1) From the explicit information (signal values at the time of events):

$$
\xi=\left\{u(t) \in C(\mathbb{R}): u\left(t_{n}\right)=x\left(t_{n}\right) \text { for all } n \in \mathbb{Z}\right\}
$$

2) From the implicit information (the value of the threshold that generated this event):

$$
\mathbb{I}=\left\{u(t) \in C(\mathbb{R}): \theta^{-} \leq u(t)<\theta^{+}(t) \text { for all } t \in \mathbb{R}\right\}
$$


3) From the knowledge that the signal is band-limited with maximum frequency $\Omega$ (Fourier decomposition of the highest order dynamics in the signals of the system):

$$
\mathbb{B}=\left\{u(t) \in \mathbb{L}^{2}(\mathbb{R}): \forall|\omega|>\Omega, \int_{-\infty}^{+\infty} u(t) e^{-j \omega t} d t=0\right\}
$$

The set $\mathbb{B}$ is convex as the band-limited signals form a linear space. For the sets $\mathbb{I}$ and $\xi$, [24] provides the proof of convexity. The reconstructed signal should be a member of the set $\xi \cap \mathbb{I} \cap \mathbb{B}$ as the constraint of the optimization. This constraint is usually a large region that makes finding the optimal solution a computation intensive task. Fortunately, because $\theta^{-}(t) \leq x(t)<\theta^{+}(t)$, one can easily derive that $\mathbb{I} \subset \xi$. Therefore, the constraint is limited to the boundary defined by $\mathbb{I} \cap \mathbb{B}$, which needs less computations for the task of real-time signal estimation.

\section{B. Projection onto Convex Sets Signal Reconstruction}

There are two methods to solve the formulated POCS problem in the literature, one-step and iterative projection. A detailed comparison of these two methods is provided in [24]. As real-time state estimation for microgrids is the aim of this paper, the later method of iterative projection onto convex sets is used, which exhibits fast computations with low precision loss. Iterative solution for POCS works by having two or more convex sets, and on each iteration the initial solution is projected to one of those convex solutions sets. By repeating the projection iteratively to those sets, the initial estimate gets closer to the optimal solution.

The projection of the signal $g$ onto a continuous convex set $C$ results in another signal $\hat{x}(t)$, which is nearest to signal $g$ :

$$
\hat{x}=P_{C g}=\arg \min _{y \in C}\|g-y\|
$$

where the projection $P_{C g}$ is closer to any $y \in C$ than $\mathrm{g}$ :

$$
\left\|P_{C g}-x\right\|<\|g-y\|
$$

For the event-based signal reconstruction problem, the initial guess $\hat{x}_{0}$ should be first projected onto convex set $\mathbb{B}$ with the following projection operator:

$$
\begin{aligned}
& P_{\mathbb{B} g}(t)=\hat{x}(t) * \frac{\Omega}{\pi} \operatorname{sinc}(\Omega t) \\
& =\int_{-\infty}^{\infty} \hat{x}(\tau) \frac{\Omega}{\pi} \operatorname{sinc}(\Omega(t-\tau)) d \tau
\end{aligned}
$$

having defined $\operatorname{sinc}(y)=\frac{\sin (y)}{y}$. $(*$ is the convolution $)$

The projection operator onto convex set $\mathbb{I}$ for clipping the signal to the boundary defined by $\theta$ is:

$$
P_{\mathbb{I g}}(t)= \begin{cases}\theta^{+}(t), & \hat{x}(t)>\theta^{+}(t) \\ \hat{x}(t), & \theta^{-}(t) \leq \hat{x}(t)<\theta^{+}(t) \\ \theta^{-}(t), & \hat{x}(t)<\theta^{-}(t)\end{cases}
$$

Finally, by applying this operator for both projections, the desired accuracy of signal reconstruction will be achieved:

$$
\hat{x}_{m+1}=P_{\mathbb{B} g} P_{\mathbb{I} g} \hat{x}_{m}, \quad m \in \mathbb{Z}
$$

The condition for stopping the projections depends on the required accuracy measures and is application dependant. By practical experiments, authors have found that 10 iterations provides an acceptable accuracy for the microgrid experiment duration, which is used in the experiment.

\section{Mean-Square Error Comparator Update Rule}

Normally, the measurements from the nodes arrive with the added noise signal. The noise is assumed to be the derivative of the Brownian motion, which is called white noise or Gaussian noise. The traditional Kalman filter is build on top of this assumption that the noise is Gaussian, however, by using the SoD sampling technique, the reconstructed signal becomes a non-Gaussian stochastic process. This leads to degradation of the estimation accuracy and longer convergence time, if it converges. Therefore, an estimator update rule is proposed here that compares the output of the Kalman filter and the reconstructed signal in real-time, and injects the correction value to the input of the Kalman filter, respectively. The correction is a dynamic offset value, which is added as described in the following:

$y_{i}\left(t_{\text {last }, i}\right)=\left\{\begin{array}{l}y_{i}(k T), \quad\left\|y_{i_{\text {predict }}}-y_{i_{\text {construct }}}<\delta\right\| \\ y_{i_{\text {construct }}}(k T), \quad\left\|y_{i_{\text {predict }}}-y_{i_{\text {construct }}} \geq \delta\right\|\end{array}\right.$

where $y_{i_{\text {predict }}}$ and $y_{i_{\text {construct }}}$ are the output of the signal reconstructor and the event-based Kalman filter, respectively.

\section{Event-Based Average Delay Consensus}

Each measurement unit, calculates the estimator communication link delay using acknowledgment round-trip delay (RTD) [29]. This value is then shared with neighbor units using the proposed event-based communication protocol. Each unit then decides the value of its SoD sampler threshold based on the average communication delay, using a linear droop mapping. In other words, when the average delay increases, the threshold for SoD sampling is also increased in order to reduce the network traffic. The droop rate can be different for the units, which provides the potential to prioritize the sampling of each unit, however for simplicity of the results comparison, a shared practical droop value is assumed in this paper. The value of the droop is tuned based on the IoT network setup of the microgrid. In the following section, the event-based average delay consensus protocol is described.

\section{A. Basic Graph Theory}

The measurement unit are connected by an undirected graph $\mathcal{G}(\mathcal{V}, \mathcal{E})$ with the nodes or vertices $\mathcal{V}=(1, \ldots, \mathcal{N})$, and the set of edges $\mathcal{E} \subset \mathcal{V} \times \mathcal{V}$. The nodes in the graph represent the measurement units, and the edges denoting the communication link between the nodes. The condition $(i, j) \in \mathcal{E}$ holds if there is a link allowing the information flow from node $i$ to node $j$ and vice versa. $A=\left[a_{i j}\right] \in R^{N \times N}$, represents the graph adjacency matrix, where $a_{i j}>0$ if $(i, j) \in \mathcal{E}$, and $a_{i j}=$ 0 otherwise. $d_{i}=\sum_{j=1}^{N} a_{i j}$ denotes the weighted degree of 
agent $v_{i}$. The degree matrix of the graph is given by $\mathbf{D}=$ $\operatorname{diag}\left\{d_{i}\right\}$, and the Laplacian matrix of the graph is derived from $\mathbf{L}=\mathbf{D}-\mathbf{A}$. An undirected graph is connected, if there exists at least one path between any two agents.

\section{B. Average Consensus Protocol}

By considering a multi-agent network with $N$ single integrator agents, the distributed average consensus will be:

$$
\dot{x}_{i}=u_{i}(t)=-\sum_{j=1}^{N} L_{i j} x_{j}(t)
$$

Since it is often not practical to have a continuous stream of data over a communication link, it is considered that each agent broadcasts its state information at specific instances (i.e., event instances) to its neighbors. Hence, we propose the following event-triggered consensus protocol:

$$
\dot{x}_{i}=u_{i}(t)=-\sum_{j=1}^{N} L_{i j} \hat{x}_{j}(t)
$$

The increasing sequence $\left\{t_{l}^{i}\right\}_{l=1}^{\infty}$ and $\left\{t_{l+1}^{i}-t_{l}^{i}\right\}_{l=1}^{\infty}$, are called the triggering times and inter-event times of agent $i$, respectively. In order to simplify the notations, let $x(t)=$ $\left[x_{1}(t), \ldots, x_{n}(t)\right]^{T}, \hat{x}(t)=\left[\hat{x}_{1}(t), \ldots, \hat{x}_{n}(t)\right]^{T}$, and $e(t)=$ $\left[e_{1}(t), \ldots, e_{n}(t)\right]^{T}=\hat{x}(t)-x(t)$. The aim is to find the correct event-triggering condition to prove the stability of the proposed consensus protocol. We state the following theorem, knowing the fact that $\lambda_{2}$ is the second smallest eigenvalue of $L$, using Lemma 1.

Lemma 1. [30] The Laplacian matrix $L$ of a connected graph $\mathbb{G}$ is positive semi-definite, i.e., $z^{T} L z \geq 0, \forall z \in \mathbb{R}^{n}$. Moreover, $z^{T} L z=0$ if and only if $z=a \mathbf{1}_{n}, a \in \mathbb{R}$, and $0 \leq \lambda_{2}(L) K_{n} \leq$ $L$, where $\lambda_{2}$ is the second smallest eigenvalue of $L$ and $K_{n}=$ $I_{n}-\frac{1}{n} 1_{n} 1_{n}^{T}$.

Theorem 1. Consider a strongly connected multi-agent directional graph with $N$ agents and the consensus protocol defined in (30). Let $0<\sigma_{i}<1$ be a constant design parameter. Given the first triggering time $t_{1}^{i}=0$, the network exponentially achieves average consensus under the eventtriggering function given as follows:

$$
e_{i}^{2}(t)-\frac{\sigma_{i}}{4 L_{i i}} \sum_{j=1}^{N} L_{i j}\left(\hat{x}_{j}(t)-\hat{x}_{i}(t)\right)^{2} \leq 0
$$

with the convergence rate, upper bounded by:

$$
\exp \left(-\frac{\left(1-\sigma_{\max }\right) \min _{i}\left\{L_{i i}\right\} \lambda_{2}(L) t}{2 \min _{i}\left\{L_{i i}\right\}+\|L\| \sigma_{\max }}\right)
$$

Proof. Following the notation of [31], $\delta(t)$ is defined as the disagreement vector with the following change of variable:

$$
x(t)=a \mathbf{1}+\delta(t)
$$

where $a$ is average of the initial state values, $a=\frac{1}{N} \sum x_{i}(t)$. Using [33], the control input of the agents will be derived as:

$$
\begin{aligned}
& \dot{\delta}(t)=-\sum_{i=1}^{N} L_{i j}\left(a+\hat{\delta}_{j}(t)\right) \\
& \left.=-a \sum_{i=1}^{N} L_{i j}-\sum_{i=1}^{N} L_{i j} \hat{\delta}_{j}(t)\right) \\
& \left.=-\sum_{i=1}^{N} L_{i j} \hat{\delta}_{j}(t)\right)
\end{aligned}
$$

Now to prove the stability, we propose the following Lyapunov function, which covers the dynamics of consensus:

$$
V(\delta(t))=\frac{1}{2} \sum_{i=1}^{N} \delta_{i}^{2} \geq 0
$$

The derivative of the Lyapunov function along the dynamic trajectory (30) will be:

$$
\begin{aligned}
& \dot{V}(\delta(t))=\sum_{i=1}^{N} \delta_{i} \dot{\delta}_{i}=\sum_{i=1}^{N} \delta_{i} \sum_{j=1}^{N}-L_{i j} \hat{\delta}_{j}(t) \\
& =\sum_{i=1}^{N}\left(\hat{\delta}_{i}-e_{i}(t)\right) \sum_{i=1}^{N}-L_{i j} \hat{\delta}_{j}(t) \\
& =\frac{1}{2} \sum_{i=1}^{N} \sum_{j=1}^{N} L_{i j}\left(\hat{\delta}_{j}(t)-\hat{\delta}_{i}(t)\right)^{2} \\
& -\sum_{i=1}^{N} \sum_{j=1}^{N} e_{i}(t) L_{i j} \hat{\delta}_{j}(t) \\
& =\frac{1}{2} \sum_{i=1}^{N} \sum_{i=1}^{N} L_{i j}\left(\hat{\delta}_{j}(t)-\hat{\delta}_{i}(t)\right)^{2} \\
& -\sum_{i=1}^{N} \sum_{j=1, j \neq i}^{N} e_{i}(t) L_{i j}\left(\hat{\delta}_{j}(t)-\hat{\delta}_{i}(t)\right)
\end{aligned}
$$

To simplify equation (36), let:

$$
\hat{f}_{i}=-\sum_{i=1}^{N} L_{i j}\left(\hat{\delta}_{j}\left(t-\tau_{i j}\right)-\hat{\delta}_{i}(t)\right)^{2}
$$

Therefore equation 36 becomes:

$$
\dot{V}(\delta(t))=\frac{1}{2} \sum_{i=1}^{N} \hat{f}_{i}-\sum_{i=1}^{N} \sum_{j=1, j \neq i}^{N} e_{i}(t) L_{i j} \hat{\delta}_{j}(t)
$$

Since $a b<a^{2}+\frac{1}{4} b^{2}, \forall a, b \in \mathbb{R}$, and

$$
\sum_{i=1}^{N} \hat{f}_{i}=-\sum_{i=1}^{N} \sum_{j=1}^{N} L_{i j}\left(\hat{\delta}_{j}\left(t-\tau_{i j}\right)-\hat{\delta}_{i}(t)\right)^{2}=\hat{\delta}^{T}(t) L \hat{\delta}(t)
$$


the following inequality holds:

$$
\begin{aligned}
& \dot{V}(\delta(t)) \leq-\frac{1}{2} \sum_{i=1}^{N} \hat{f}_{i}-\sum_{i=1}^{N} \sum_{j=1, j \neq i}^{N} L_{i j} e_{i}^{2}(t) \\
& -\sum_{i=1}^{N} \sum_{j=1, j \neq i}^{N} L_{i j} \frac{1}{4}\left(\hat{\delta}_{j}\left(t-\tau_{i j}\right)-\hat{\delta}_{i}(t)\right)^{2} \\
& =-\frac{1}{4} \sum_{i=1}^{N} \hat{f}_{i}+\sum_{i=1}^{N} L_{i j} e_{i}^{2}(t)
\end{aligned}
$$

From (31) and 40), we have:

$$
\begin{aligned}
& \dot{V}(\delta(t)) \leq-\frac{1}{4} \sum_{i=1}^{N} \hat{f}_{i}+\sum_{i=1}^{N} L_{i j} e_{i}^{2}(t) \\
& \leq-\frac{1}{2}\left(1-\sigma_{\text {max }}\right) \hat{\delta}^{T}(t) L \hat{\delta}(t)
\end{aligned}
$$

where $\sigma_{\max }=\max \left\{\sigma_{1}, \ldots, \sigma_{n}\right\}$. In addition, we have:

$$
\begin{aligned}
& \delta^{T}(t) L \delta(t)=(\hat{\delta}(t)+e(t))^{T} L(\hat{\delta}(t)+e(t)) \\
& \leq 2 \hat{\delta}^{T}(t) L \hat{\delta}+2 e^{T}(t) L e(t) \\
& \leq 2 \hat{\delta}^{T}(t) L \hat{\delta}+\frac{\|L\| \sigma_{\max }}{2 \min _{i}\left\{L_{i i}\right\}} \sum_{i=1}^{N} \hat{f}_{i} \\
& =\left(2+\frac{\|L\| \sigma_{\max }}{2 \min _{i}\left\{L_{i i}\right\}}\right) \hat{\delta}^{T}(t) L \hat{\delta}
\end{aligned}
$$

where (42) holds because $L$ is a positive semi-definite matrix and $2 a^{T} L b \leq a^{T} L a+b^{T} L b, \forall a, b \in \mathbb{R}^{n}$ and 43 holds since (31) and $a^{T} L a \leq\|L\|\|a\|^{2}, \forall a \in \mathbb{R}^{n}$. Finally we have:

$$
\begin{aligned}
& \dot{V}(\delta(t)) \leq-\frac{\left(1-\sigma_{\max }\right) \min _{i}\left\{L_{i i}\right\}}{4 \min _{i}\left\{L_{i i}\right\}+2\|L\| \sigma_{\max }} \delta^{T}(t) L \delta(t) \\
& \leq-\frac{\left(1-\sigma_{\max }\right) \min _{i}\left\{L_{i i}\right\} \lambda_{2}(L)}{2 \min _{i}\left\{L_{i i}\right\}+1\|L\| \sigma_{\max }} V(\delta(t))
\end{aligned}
$$

(45) holds due to Lemma 1, hence:

$$
\dot{V}(\delta(t)) \leq V(\delta(0)) \exp \left(-\frac{\left(1-\sigma_{\max }\right) \min _{i}\left\{L_{i i}\right\} \lambda_{2}(L) t}{2 \min _{i}\left\{L_{i i}\right\}+\|L\| \sigma_{\max }}\right) .
$$

This shows that the multi-agent system 30 with eventtriggering condition 31 exponentially reaches stability, as long as $\mathcal{G}$ is connected.

\section{Analysis of the Observer Optimality}

The optimality of the proposed solution can be analysed from several aspects. From the state estimation aspect, the employed Kalman state estimator is known to be an optimal linear state estimator in the literature of state estimation. It follows from theory that the Kalman filter is the optimal linear filter in cases where:

- The model perfectly matches the real system

- The measurement noise is white (uncorrelated)

- The covariances of the noise are exactly known

When a Kalman filter works optimally, the update sequence (the output prediction error) is white noise, therefore the whiteness property of the updates defines the estimation performance. The existence, optimality and stability of the Kalman filter with partial observations are discussed in [32].
The proposed POCS signal re-constructor is a recursive method that converges to a convex set consisting of the original signal after several iterations. The reconstructed signal found by the POCS method, is an optimal solution that can be build from the SoD generated samples. The convergence to the solution is proved in [33] along with the analysis on the convergence rate. From the communication and channel utilization aspect, the solution is optimal compared to the traditional time-based sampling technique such as Zero-orderhold $(\mathrm{ZOH})$. In the proposed solution, the network packet is only generated when there is a deviation from the $\mathrm{SoD}$ threshold or the average consensus protocol requires a new data-sharing event.

\section{Vili. Estimator Validation Results}

In order to evaluate the state estimator performance, an IoT-based setup is designed that consists of IoT smart meters based on Seeeduino ${ }^{\circledR}$ Dragon IoT evaluation boards and a DC microgrid real-time simulator from dSPACE ${ }^{\circledR}$ (SCALEXIO Real-Time Simulator). Each node has a long range wide area network (LoRaWAN) communication module and supports IEEE $802.11 \mathrm{~b} / \mathrm{g} / \mathrm{n}$ (WiFi) communication protocol. The nodes are interfaced to the real-time microgrid simulator via an interface stackable shield that can be measure analog input and output signals. The setup is shown in Fig. 2 The proposed SoD sampling strategy is implemented using digital signal processing instructions of ARM Cortex M0+, and the microgrid model is implemented by using MATLAB/Simulink real-time code generator. WiFi protocol necessitates a router gateway to be used for the data collection. In this setup, a Raspberry Pi computer with the supporting communication modules for the gateway operation is used. This gateway receives the data from the measurement nodes via MQTT (Message Queuing Telemetry Transport) protocol. Thingsboard ${ }^{\circledR}$ software implements the MQTT broker, which is used for data archiving and processing. By using the mentioned protocols and devices, the microgrid monitoring cost can be considerably cheaper than other smart metering technologies such as IEC 61850 [34]. The state estimator was implemented on the real-time microgrid simulator. The nodes measure the signals and transmit them over the wireless network to the real-time simulator. Therefore, all of the results are observed at the realtime simulator. The IEEE 5 bus reference microgrid with the nominal $110 \mathrm{~V}$ bus voltage and $10 \mathrm{~kW}$ reference power for per-unit calculations, as shown in Fig. 3, is chosen for the case study. The droop controllers are designed based on the technique proposed in [1]. The $Y$ bus admittance matrix for state representation of the microgird is derived based on the line parameters in Table I] Also the covariance parameters and initial SoD threshold of the estimator is shown in Table III. The IEEE 5 bus microgrid system is standardized with a set of reference line parameters which includes the resistance, reactance, and the susceptance of the lines based on $50 \mathrm{~Hz}$ $\mathrm{AC}$ frequency. In a DC microgrid, the values of resistance, inductance, and capacitance are the important line dynamics required to be modelled in the simulation. Therefore, we have converted the corresponding values in the AC system to their 


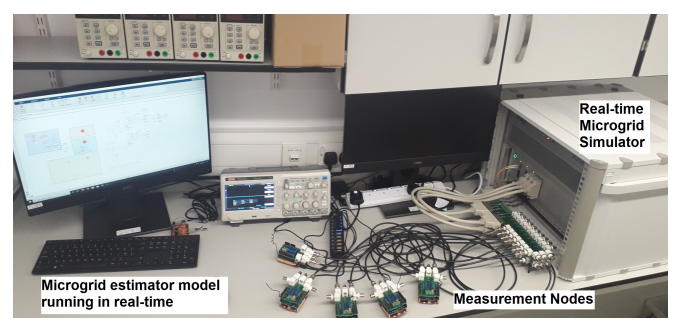

Fig. 2. Experimental setup for evaluation of the proposed estimator.

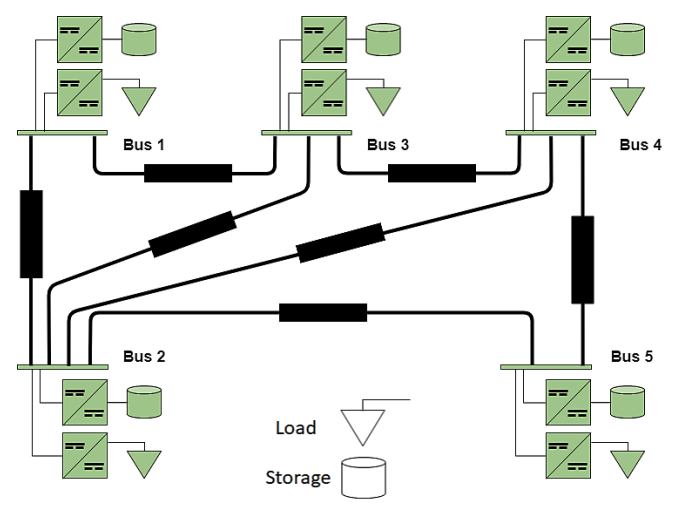

Fig. 3. IEEE 5 bus case study for estimator validation.

TABLE I

LINE PARAMETERS OF THE IEEE 5 BUS MICROGRID .

\begin{tabular}{|c|c|c|}
\hline Line & Line Impedance (p.u.) & Line Susceptance (p.u) \\
\hline $1-2$ & $0.02+j 0.06$ & $j 0.03$ \\
\hline $1-3$ & $0.08+j 0.24$ & $j 0.025$ \\
\hline $2-3$ & $0.06+j 0.25$ & $j 0.02$ \\
\hline $2-4$ & $0.06+j 0.18$ & $j 0.02$ \\
\hline $2-5$ & $0.04+j 0.12$ & $j 0.015$ \\
\hline $3-4$ & $0.01+j 0.03$ & $j 0.01$ \\
\hline $4-5$ & $0.08+j 0.24$ & $j 0.025$ \\
\hline
\end{tabular}

equivalent DC system with the following simple formula in per-unit: $R_{d c}=R_{a c}$ (not considering the corona effect), $L_{d c}=\frac{X_{L}}{2 * \pi * f}$, and $C_{d c}=\frac{2 * \pi * f}{X_{C}}$

The simulations is run for 5 seconds, and the estimator converges to the actual state in 600 milliseconds, as shown in Fig. 4 Furthermore, Fig. 5 shows that the proposed estimation strategy has achieved a better performance comparing to the traditional Kalman filter by converging to lower steady state error. For this simulation, the traditional Kalman filter runs in a digital platform with 0.1 millisecond period, needs 10,000 events in 1 second to achieve the same performance as our proposed estimation strategy, with only a few hundred events. The Kalman filter has always been challenged for its high speed measurement requirements, however the proposed estimator has opened new doors for event-based state estimators with low communication speed requirements. The event generation density over the time is shown in Fig. 6. Also as shown in Fig. 8, the steady state estimation error is comparatively lower comparing to the classic Kalman filter. The comparison of the observer methods are provided in Table IIII From the network traffic perspective, Fig. 9 illustrates the comparison of

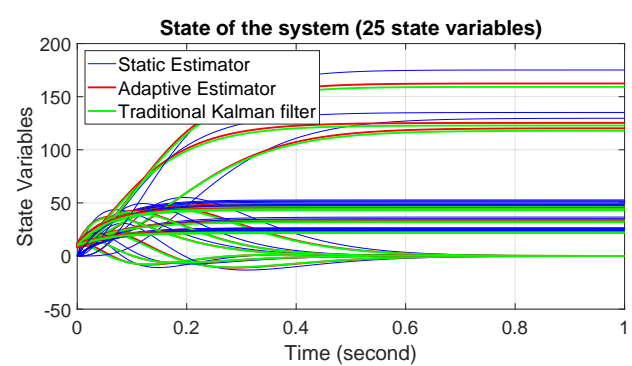

Fig. 4. Experiment result 1: State variables of the microgrid. The microgrid is realized into 25 state variables that need to be estimated by the proposed observer.

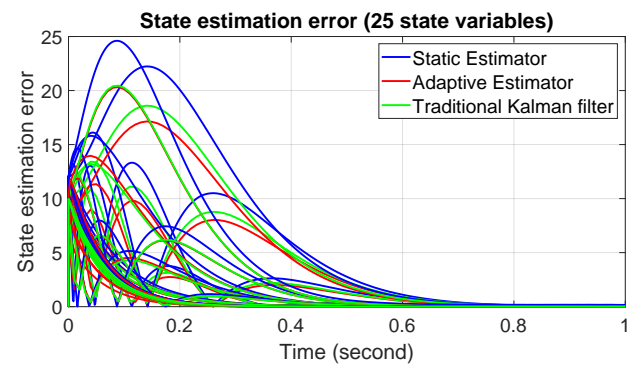

Fig. 5. Experiment result 2: The estimation error of the observers for comparison. The static estimator has a constant threshold with the value "1", and the adaptive estimator is initialized with the same value.

the proposed adaptive SoD sampler estimator with the static threshold state estimator. The accumulative number of packets transmitted in the network, is reduced more than $40 \%$ from the static threshold SoD sampling method. Also, the existence of the threshold, guarantees that the Zeno behaviour will never happen, as can be seen in Fig. 6 During the experimental, it was found that the LoRaWAN communication protocol has significant limitations, which can decrease the accuracy of estimation. It introduces a large value of delay between the events transmission in the range of seconds, especially when the number of messages per unit of time gets higher than the capacity of the network. The threshold of the SoD sampler directly affects the amount of messages, therefore a tuning algorithm will be required in order to make a relation between the estimation error, sampling threshold, and the number of events. Nevertheless, by using a high speed WiFi communication network, the ideal performance was achieved, fulfilling the data collection strategy requirements.

TABLE II

STATE ESTIMATOR PARAMETERS FOR SIMULATION.

\begin{tabular}{|c|c|}
\hline$\delta(0)$ (threshold initial value) & 1 \\
\hline$Q$ (Process Noise Covariance) & 0.1 \\
\hline$R$ (Measurement Noise Covariance) & 0.36 \\
\hline$T$ (Estimator Sampling Time) & 100 microseconds \\
\hline$\sigma_{\max }$ (Even generation parameter) & 0.6 \\
\hline
\end{tabular}

\section{Concluding Remarks}

In this paper, an event-based optimal observer is proposed for the microgrids. The proposed estimator works based on 
TABLE III

COMPARISON OF THE OBSERVERS BY THEIR REQUIRED NETWORK CHARACTERISTICS.

\begin{tabular}{|c|c|c|c|c|c|}
\hline Observer & Sampling Type & Communication Delay & Resource Usage & Operation Type & Accuracy \\
\hline Traditional (Classic) Kalman Filter 132 & Periodic & Not considered & High & Centralized & High \\
\hline Static Threshold SoD-Based Kalman Filter 23 & Event-based & Not considered & Low & Centralized & Medium \\
\hline Proposed Adaptive Observer with POCS Conditioner & Event-based & Adaptive & Medium & Distributed & High \\
\hline
\end{tabular}

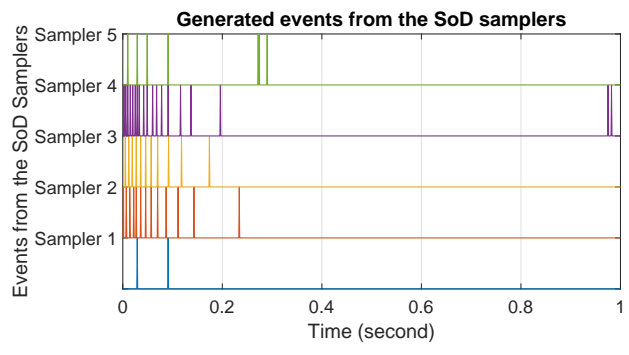

Fig. 6. Experiment result 3: Event generation density from the adaptive SoD sampler nodes. As can be seen, the number of generated events gets decreased as the system enters into its steady state mode.

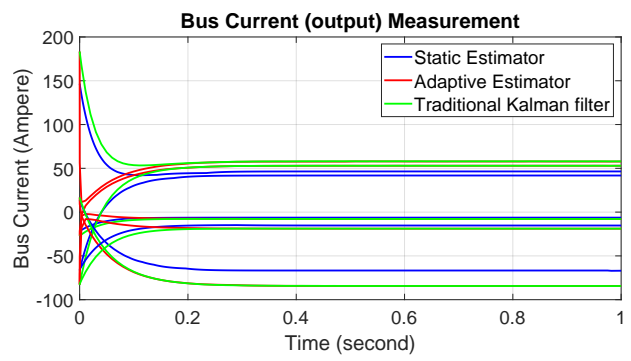

Fig. 7. Experiment result 4: The injected currents of the buses (system outputs). The nominal voltage is $110 \mathrm{~V}$. Negative current means power generation and positive current means consumption at the buses.

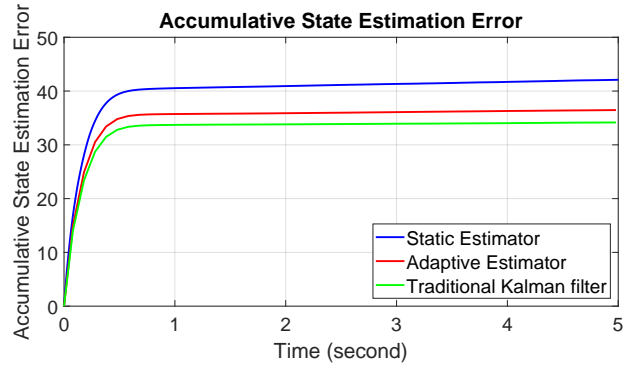

Fig. 8. Experiment result 5: Accumulative estimation error comparison between the traditional Kalman filter, the proposed adaptive threshold state estimator, and the static state estimator.

send-on-delta (SoD) non-uniform sampling method and furthermore, the SoD threshold is adaptive with regard to the average communication delay. The average delay is decided using the event-based average consensus protocol. The estimation error is further corrected by projection onto convex sets algorithm to have a higher estimation accuracy. It was resulted that the estimator has low estimation error comparing to the classic Kalman filter, with only a few events exchanged in the communication network. The optimality of the solution is analysed along with a step by step design procedure. The performance of the observer is studied in the reference IEEE 5

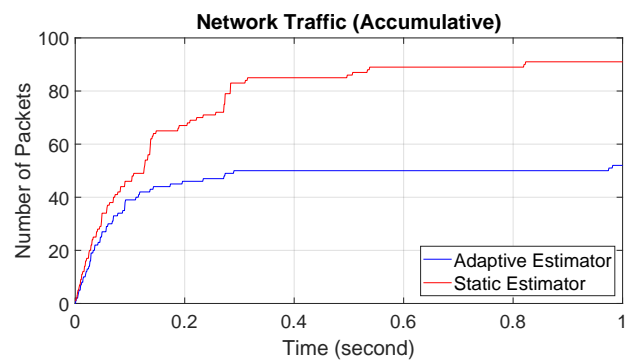

Fig. 9. Experiment result 6: Network traffic comparison between the static SoD sampler and the proposed adaptive strategy. It can be seen that the overall traffic is reduced by more than $40 \%$.

Bus microgrid. For the future study, the results can be extended to consider the packet drop out in the observer performance.

\section{REFERENCES}

[1] S. A. Alavi, K. Mehran, Y. Hao, A. Rahimian, H. Mirsaeedi, and V. Vahidinasab, "A distributed event-triggered control strategy for dc microgrids based on publish-subscribe model over industrial wireless sensor networks," IEEE Transactions on Smart Grid, vol. 10, no. 4, pp. 4323-4337, 2019.

[2] S. Parhizi, H. Lotfi, A. Khodaei, and S. Bahramirad, "State of the art in research on microgrids: A review," IEEE Access, vol. 3, pp. 890-925, 2015.

[3] B. Qin, H. Sun, J. Ma, W. Li, T. Ding, Z. Wang, and A. Y. Zomaya, "Robust control of doubly fed wind generator via state-dependent riccati equation technique," IEEE Transactions on Power Systems, vol. 34, no. 3, pp. 2390-2400, 2019.

[4] S. A. Alavi, M. Javadipour, and K. Mehran, "State Monitoring for Situational Awareness in Rural Microgrids Using the IoT Infrastructure," arXiv e-prints, Jun. 2019.

[5] E. Ghahremani and I. Kamwa, "Local and wide-area PMU-based decentralized dynamic state estimation in multi-machine power systems," IEEE Transactions on Power Systems, vol. 31, no. 1, pp. 547-562, Jan. 2016.

[6] M. H. Amini, H. Arasteh, and P. Siano, "Sustainable smart cities through the lens of complex interdependent infrastructures: Panorama and stateof-the-art," in Studies in Systems, Decision and Control. Springer, Cham, 2019, vol. 186, pp. 45-68.

[7] S. Amir Alavi, A. Rahimian, K. Mehran, and J. Alaleddin Mehr Ardestani, "An IoT-Based Data Collection Platform for Situational Awareness-Centric Microgrids," in Canadian Conference on Electrical and Computer Engineering, vol. 2018-May. IEEE, 2018, pp. 1-4.

[8] A. Primadianto and C. N. Lu, "A Review on Distribution System State Estimation," IEEE Transactions on Power Systems, vol. 32, no. 5, pp. 3875-3883, Sep. 2017.

[9] H. Li, L. Lai, and W. Zhang, "Communication requirement for reliable and secure state estimation and control in smart grid," IEEE Transactions on Smart Grid, vol. 2, no. 3, pp. 476-486, 2011.

[10] Z. Akhtar, S. Amir Alavi, and K. Mehran, "Voltage Control in LV Networks Using Electric Springs with Coordination," in Canadian Conference on Electrical and Computer Engineering, vol. 2018-May. Institute of Electrical and Electronics Engineers Inc., Aug. 2018, pp. $1-5$.

[11] A. Abessi, V. Vahidinasab, and M. S. Ghazizadeh, "Centralized support distributed voltage control by using end-users as reactive power support," IEEE Transactions on Smart Grid, vol. 7, no. 1, pp. 178-188, Jan. 2016. 
[12] S. Bhela, V. Kekatos, and S. Veeramachaneni, "Enhancing observability in distribution grids using smart meter data," IEEE Transactions on Smart Grid, vol. 9, no. 6, pp. 5953-5961, Nov. 2018.

[13] A. Anta and P. Tabuada, "To sample or not to sample: Self-triggered control for nonlinear systems," IEEE Transactions on Automatic Control, vol. 55, no. 9, pp. 2030-2042, Sep. 2010.

[14] A. Alimardani, F. Therrien, D. Atanackovic, J. Jatskevich, and E. Vaahedi, "Distribution System State Estimation Based on Nonsynchronized Smart Meters," IEEE Transactions on Smart Grid, vol. 6, no. 6, pp. 2919-2928, Nov. 2015.

[15] M. Pau, E. Patti, L. Barbierato, A. Estebsari, E. Pons, F. Ponci, and A. Monti, "Design and Accuracy Analysis of Multilevel State Estimation Based on Smart Metering Infrastructure," IEEE Transactions on Instrumentation and Measurement, vol. 68, no. 11, pp. 4300-4312, Nov. 2019.

[16] M. M. Rana, L. Li, and S. Su, "Distributed State Estimation Using RSC Coded Smart Grid Communications," IEEE Access, vol. 3, pp. 13401349, 2015.

[17] S. M. Alam, B. Natarajan, and A. Pahwa, "Distribution grid state estimation from compressed measurements," IEEE Transactions on Smart Grid, vol. 5, no. 4, pp. 1631-1642, Jul. 2014.

[18] E. B. Alzate, M. Bueno-López, J. Xie, and K. Strunz, "Distribution System State Estimation to Support Coordinated Voltage-Control Strategies by Using Smart Meters," IEEE Transactions on Power Systems, vol. 34, no. 6, pp. 5198-5207, Nov. 2019.

[19] Y. Weng, R. Negi, C. Faloutsos, and M. D. Ilic, "Robust Data-Driven State Estimation for Smart Grid," IEEE Transactions on Smart Grid, vol. 8, no. 4, pp. 1956-1967, Jul. 2017.

[20] D. Trihinas, G. Pallis, and M. D. Dikaiakos, "AdaM: An adaptive monitoring framework for sampling and filtering on IoT devices," in 2015 IEEE International Conference on Big Data (Big Data). IEEE, Oct. 2015 , pp. $717-726$.

[21] L. Fan and L. Xiong, "Adaptively Sharing Time-Series with Differential Privacy," Feb. 2012.

[22] D. Zucchetto, C. Pielli, A. Zanella, and M. Zorzi, "Random Access in the IoT: An Adaptive Sampling and Transmission Strategy," in 2018 IEEE International Conference on Communications (ICC), vol. 2018May. IEEE, May 2018, pp. 1-6.

[23] S. A. Alavi, M. Javadipour, and K. Mehran, "Microgrid Optimal State Estimation Over IoT Wireless Sensor Networks With Event-Based Measurements," in IECON 2019 - 45th Annual Conference of the IEEE Industrial Electronics Society. IEEE, Oct. 2019, pp. 4145-4150.

[24] D. Rzepka, M. Miskowicz, D. Koscielnik, and N. T. Thao, "Reconstruction of signals from level-crossing samples using implicit information," IEEE Access, vol. 6, pp. 35001-35011, 2018.

[25] W. Li, Y. Jia, and J. Du, "Event-triggered Kalman consensus filter over sensor networks," IET Control Theory and Applications, vol. 10, no. 1, pp. 103-110, Jan. 2016

[26] A. Zakhor and A. V. Oppenheim, "Reconstruction of Two-Dimensional Signals from Level Crossings," Proceedings of the IEEE, vol. 78, no. 1, pp. 31-55, 1990.

[27] Y. H. Fung and Y. H. Chan, "A POCS-based restoration algorithm for restoring halftoned color-quantized images," IEEE Transactions on Image Processing, vol. 15, no. 7, pp. 1985-1992, Jul. 2006.

[28] S.-j. Yeh and H. Stark, "Iterative and one-step reconstruction from nonuniform samples by convex projections," Journal of the Optical Society of America A, vol. 7, no. 3, p. 491, Mar. 1990.

[29] S. Huang, L. Zhao, M. Jiang, and W. Liu, "Improved preamble detection and round-trip delay estimation for random access in high-mobility airborne communication systems," in 2019 IEEE/CIC International Conference on Communications in China, ICCC 2019, Aug. 2019, pp. 384-388.

[30] M. Mesbahi and M. Egerstedt, Graph theoretic methods in multiagent networks. Princeton University Press, 2010.

[31] R. Olfati-Saber and R. M. Murray, "Consensus problems in networks of agents with switching topology and time-delays," IEEE Transactions on Automatic Control, vol. 49, no. 9, pp. 1520-1533, 2004.

[32] J. Su, B. Li, and W. H. Chen, "On existence, optimality and asymptotic stability of the Kalman filter with partially observed inputs," Automatica, vol. 53, pp. 149-154, Mar. 2015.

[33] F. Deutsch and H. Hundal, "The rate of convergence for the cyclic projections algorithm III: Regularity of convex sets," Journal of Approximation Theory, vol. 155, no. 2, pp. 155-184, Dec. 2008.

[34] M. Iglesias-Urkia, D. Casado-Mansilla, S. Mayer, J. Bilbao, and A. Urbieta, "Integrating Electrical Substations Within the IoT Using IEC 61850, CoAP, and CBOR," IEEE Internet of Things Journal, vol. 6, no. 5, pp. 7437-7449, Oct. 2019.

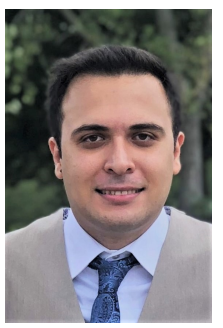

Seyed Amir Alavi (GS'16) received the B.Sc. and M.Sc. degrees in electrical engineering from Power and Water University of Technology (PWUT), Tehran, Iran, and Shahid Beheshti University (SBU), Tehran, Iran, in 2013 and 2017, respectively. He is currently pursuing the Ph.D. degree in electronic engineering at Queen Mary University of London (QMUL). He has conducted a number of industrial projects on implementation of smart energy management services based on loT solutions. As an embedded system designer, he has given consultancy to multiple international companies and projects in Iran and UK. His research interests include embedded control systems, microgrids, event-based control and signal processing, networked control systems, and wireless sensor networks.

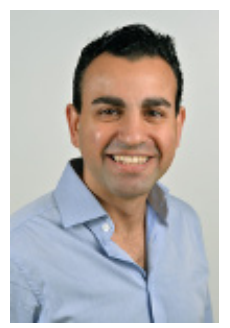

Kamyar Mehran (M'09, SM'20) is a Senior Lecturer (Associate Professor) in Electrical Power Engineering and the director of the Real-Time Power and Control System (RPCS) Laboratory at the school of EECS in Queen Mary University in London. He received his $\mathrm{PhD}$ degree in Electrical and Electronic Engineering and MSc degree in Control and Automation from Newcastle University, UK, in 2010 and 2004 respectively His current research is mainly focused on the theory and application of advanced control and optimisation methods for (vehicular, grid) energy storage, SiC-based power electronics and dc/ac Microgrids and Nanogrids with over 40 peer-reviewed journal publications, and several book chapters including his recent chapter in "Control strategy for time-delay systems" published by Elsevier. Dr Mehran attracted £1.27M external funding in his academic career as a principal investigator and co-investigator from the UK research and innovation (UKRI) councils including EPSRC, Innovate UK and the prestigious global challenge funding from Royal Society and Newton fund. He is a reviewer for the EPSRC and several highimpact IEEE Transactions journals including IEEE Transactions in Fuzzy Systems and IEEE Transactions in Smart Grid. He has been an invited speaker in several conferences and companies. From 2013 to 2015, he was a research fellow in the University of Warwick, UK and from 20102013 he was a research associate in the Newcastle University where he also acted as a commercialisation manager for a university spinoff company. Before his academic career, he worked for eight years in industry in a variety of software-related roles.

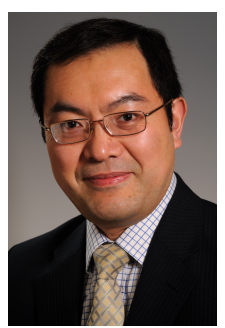

Yang Hao (M'00-SM'06-F'13) received the Ph.D. degree in computational electromagnetics from the Centre for Communications Research, University of Bristol, Bristol, U.K., in 1998. He was a Postdoctoral Research Fellow with the School of Electronic, Electrical and Computer Engineering, University of Birmingham, Birmingham, U.K. $\mathrm{He}$ is currently a Professor of antennas and electromagnetics with the Antenna Engineering Group, Queen Mary University of London, London, U.K. His work has been recognised both nationally and internationally through his books "Antennas and Radio Propagation for Body-Centric Wireless Communications" and "FDTD Modeling of Metamaterials: Theory and Applications," (Artech House, USA) and highly cited papers published in leading journals, including Nature Group Publications, Physical Review Letters, Applied Physics Letters, IEEE proceedings, and transactions. Prof. Hao won many accolades, including the prestigious AF Harvey Prize in 2015, the BAE Chairman's Silver Award in 2014, and the Royal Society Wolfson Research Merit Award in 2013. Internationally, he has achieved his reputation for his leadership in IEEE, being an Editor-in-Chief for IEEE ANTENNAS AND WIRELESS PROPAGATION LETTERS from 2013 to 2017 and founding a new open access journal EPJ Applied Prof. Hao is an elected Fellow of Royal Academy of Engineering, the Institute of Electrical and Electronics Engineers (IEEE) and the Institution of Engineering and Technology (IET). 